\title{
Active Magnetic Bearing Rotor Model Updating Using Resonance and MAC Error
}

\author{
Yuanping Xu, ${ }^{1}$ Jin Zhou, ${ }^{1}$ Long $\mathrm{Di}^{2}{ }^{2}$ Chen $\mathrm{Zhao}^{1}{ }^{\text {and }}$ Qintao Guo ${ }^{1}$ \\ ${ }^{1}$ College of Mechanical and Electrical Engineering, Nanjing University of Aeronautics and Astronautics, Nanjing 210016, China \\ ${ }^{2}$ Charles L. Brown Department of Electrical and Computer Engineering and Rotating Machinery and Control Laboratory (ROMAC), \\ University of Virginia, P.O. Box 400473, Charlottesville, VA 22904-4743, USA \\ Correspondence should be addressed to Jin Zhou; zhj@nuaa.edu.cn
}

Received 10 October 2014; Accepted 15 December 2014

Academic Editor: Brij N. Agrawal

Copyright (C) 2015 Yuanping Xu et al. This is an open access article distributed under the Creative Commons Attribution License, which permits unrestricted use, distribution, and reproduction in any medium, provided the original work is properly cited.

\begin{abstract}
Modern control techniques can improve the performance and robustness of a rotor active magnetic bearing (AMB) system. Since those control methods usually rely on system models, it is important to obtain a precise rotor AMB analytical model. However, the interference fits and shrink effects of rotor AMB cause inaccuracy to the final system model. In this paper, an experiment based model updating method is proposed to improve the accuracy of the finite element (FE) model used in a rotor AMB system. Modelling error is minimized by applying a numerical optimization Nelder-Mead simplex algorithm to properly adjust FE model parameters. Both the error resonance frequencies and modal assurance criterion (MAC) values are minimized simultaneously to account for the rotor natural frequencies as well as for the mode shapes. Verification of the updated rotor model is performed by comparing the experimental and analytical frequency response. The close agreements demonstrate the effectiveness of the proposed model updating methodology.
\end{abstract}

\section{Introduction}

As potential alternatives to conventional mechanical bearings, active magnetic bearings have been increasingly used in compressors, pumps, and many other high-speed rotating machineries [1]. AMBs support rotors by using electromagnetic field rather than mechanical forces as in lubricated fluid films or contact of rolling element bearings [2]. AMBs possess several advantages over mechanical bearings such as low friction, no need for lubrication, and high attainable rotating speed.

The AMB system is open loop unstable and feedback control is needed for levitation. This function is commonly achieved by single input and single output (SISO) controller design such as proportional-integral-derivative (PID) controller [3]. However, decentralized PID controllers do not always provide the desired performance and robustness especially when the operating speed is above the system's critical speed and the gyroscopic effect is involved. In order to enhance the robustness, modern control techniques such as $H_{\infty}$ and $\mu$ synthesis have been applied to rotor $\mathrm{AMB}$ system. Since these multiple input and multiple output (MIMO) control methods usually rely on system models, it is important to obtain a precise rotor AMB analytical model and obtaining an analytical model close to the actual system is an inevitable step before the controller design $[4,5]$.

For a rotor $\mathrm{AMB}$ system, its dynamic properties play a key role. The rotor model is often obtained by the finite element method (FEM) or the transfer matrix method. For a beam type structure, the transfer matrix and the FEM formulations are theoretically equivalent, but the FEM formulation is usually more numerically stable [6]. For rotor AMB system, the rotor dynamic characteristics such as resonance and mode shape calculated by the rotor model are not always equivalent to the experimental results since the rotor adopted in $\mathrm{AMB}$ system is assembled by several components with interference fits. When assembled, the resulting AMB rotor exhibits inhomogeneous material properties which are assumed as a source of modelling error [7]. For example, a typical rotor AMB system includes rotor shaft, front/rear AMB lamination 
stacks, thrust AMB lamination stacks, sensor rings, and motor (Figure 2), which are assembled with interference fits and shrink effects, contributing to the bending stiffness of the rotor shaft [8].

Dynamic modelling is an important consideration for structure design and almost always results in errors to some degree when compared to experimental result [9]. The difference between experimental data and finite element models is generated for various reasons which primarily originate from unknown structure features and simplifications $[7,10]$. Finite element model updating techniques are used to adjust selected parameters of models to minimize the discrepancy and establish a model which is more compatible with experimental data and more closely represents the dynamic characteristic of the object [11]. These techniques have been widely applied in aerospace, heavy equipment, automobiles, construction, and other engineering fields [12]. The direct and iterative model updating methods have been applied in both time and frequency domains to perform model updating [13]. The direct methods modify the system mass, stiffness, and/or damping matrix to produce the desired results. However, the drawbacks of this approach are that the updated mathematical representation may not be physically meaningful, which in many cases is not acceptable. In contrast, iterative methods select the updating parameters flexibly and provide meaningful updated results. Iterative methods need an objective or error function to represent the discrepancy in frequency response such as resonance frequency, antiresonance frequency, MAC, or mode shapes between experimental and theoretical results. Iterations are terminated when satisfactory accuracy is reached. This approach commonly employs optimization routines which minimize an error function [7].

Although model updating approaches have been widely applied to other engineering field, few literatures can be found in applying model updating to rotor AMB system. Li et al. [2] set the first five modal frequencies of the freefree rotor model as the error function and updated eight uncertain stiffness factors, but [2] only considered the modal frequencies without providing the mathematical mode shape, so the mode shape information is not available for the controller design. Considering the shrink fit effect, Wroblewski [7] set the first three resonance and antiresonance frequency points of AMB system open loop transfer function as the error function and updated fifteen moduli of elasticity using Nelder-Mead nonlinear unconstrained optimization method. After updating the rotor AMB model, Wroblewski validated the results by designing a $\mu$ synthesis controller based on the updated model and the experimental results show that the rotor levitates robustly.

The MAC is widely used to evaluate the comparison of mode shapes. In this paper, after building a rotor finite element model, we update the rotor model using NelderMead nonlinear unconstrained optimization method and provide an objective function, combining first four MAC values and bending frequency errors together. The advantages of this combination are that the mode shapes calculated by the updated model are highly accurate as well as the normal modal frequencies. By comparing the updated
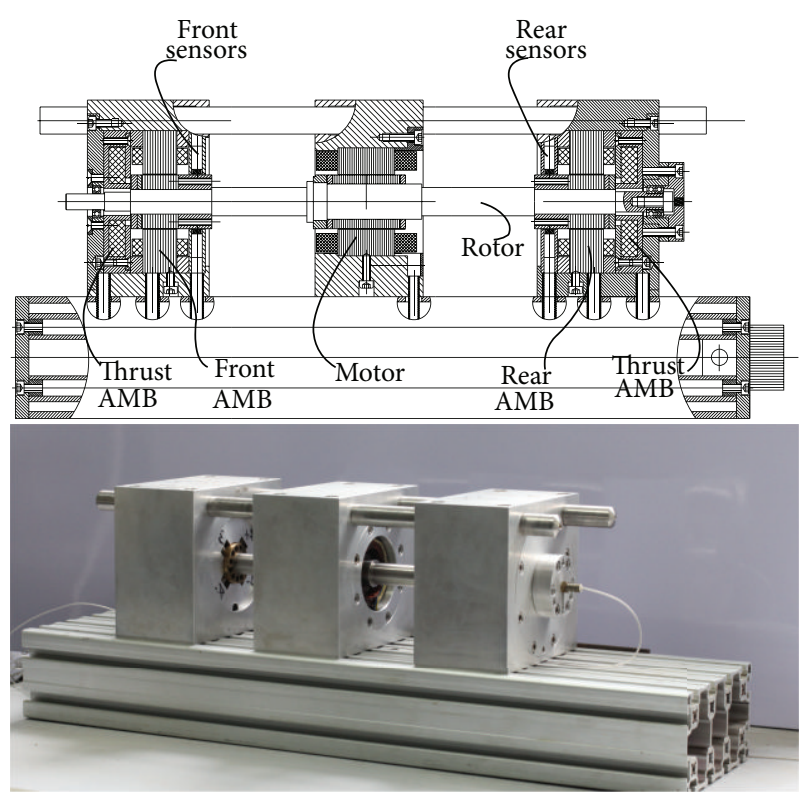

FIgURE 1: An overview of the rotor AMB test rig.

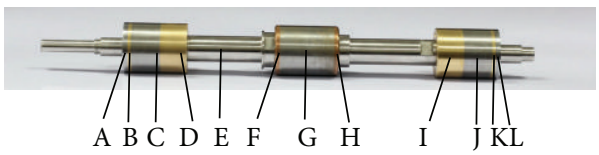

FIGURE 2: Rotor of the AMB system.

model's dynamic characteristics with experimental data, the close agreements demonstrate that the proposed method is effective for AMB rotor model updating.

The remainder of the paper is organized as follows. Section 2 describes the rotor AMB test rig. Section 3 presents the mathematical modelling of AMB rotor and model updating approach using resonance frequencies and MAC as error function. Section 4 presents the verification of the updated rotor model by comparing the experimental and theoretical frequency responses. Conclusions are drawn in Section 5.

\section{Rotor AMB Test Rig}

The experimental test rig for this study is five degrees of freedom (DOFs) rotor AMB system designed and built as a research platform at Nanjing University of Aeronautics and Astronautics, pictured in Figure 1. The rotor is supported by two radial and two thrust $\mathrm{AMBs}$ and is designed to have a maximum speed of $60,000 \mathrm{rpm}$. Unlike other rotor $\mathrm{AMB}$ systems where the thrust AMB is located between front and rear radical $A M B s$, the thrust $A M B$ here is integrated with radical $A M B$, which makes it easy to assemble and disassemble. The AMB rotor is $0.468 \mathrm{~m}$ long and weighs around $2.4 \mathrm{~kg}$ as illustrated in Figure 2. A $1.5 \mathrm{~kW}$ AC asynchronous induction motor rotor is located in the middle of the rotor between the front AMB and rear AMB. Two laminated silicon steel sheets are mounted at the end of rotor shaft for the two radical support AMBs and one laminated silicon steel sheet 
TABLE 1: Specific details of the rotor.

\begin{tabular}{lc}
\hline Label & Name \\
\hline A/L & Front/rear thrust AMB lamination stacks \\
B/K & Magnetic isolation ring \\
$\mathrm{C} / \mathrm{J}$ & Front/rear radical AMB lamination stacks \\
$\mathrm{D} / \mathrm{I}$ & Front/rear sensor ring \\
$\mathrm{E}$ & Rotor shaft \\
$\mathrm{F} / \mathrm{H}$ & Motor rotor fix stacks \\
$\mathrm{G}$ & Motor rotor \\
\hline
\end{tabular}

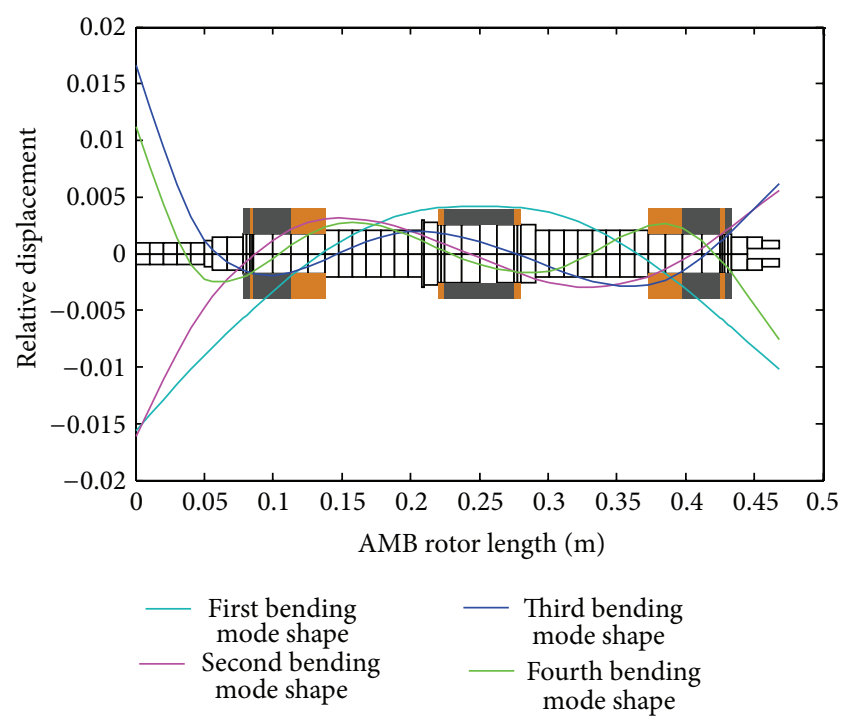

FIgURE 3: Theoretic mode shapes of free-free rotor.

is assembled in the middle for the driven motor. The specific details of the rotor are listed in Table 1. The radical and axial air gaps are $0.3 \mathrm{~mm}$ and $0.5 \mathrm{~mm}$, respectively. Two rolling element ball bearings are assembled at the radical support $\mathrm{AMB}$ casings to prevent damage to $\mathrm{AMBs}$ when the rotor drops.

\section{Rotor Modelling and Model Updating}

3.1. Finite Element Model. The nominal rotor finite element model has been created using beam finite elements. Previous experiences have confirmed that relatively simple beam element models are adequate for analyzing most rotors. Beam finite elements used currently have two or three nodes per element, with two nodes being most common since they are easy to model typical industrial rotor geometries. With four DOFs at each node it enables the simultaneous modelling of beam deflection in both horizontal and vertical planes [8].

The rotor finite element model is developed according to the geometrical and mass information, pictured in Figure 3. 53 beam elements, including 54 nodes, are used to model the rotor. The assembled parts such as front and rear AMB lamination stacks are modelled as lumped mass onto the corresponding nodes. Ignoring two DOFs in axial direction, each node contains 4 radical DOFs, 2 translational and 2
TABLE 2: Experimental and theoretical (no updating) modal frequencies.

\begin{tabular}{lccc}
\hline $\begin{array}{l}\text { Bending } \\
\text { modes }\end{array}$ & $\begin{array}{c}\text { Calculated } \\
\text { freq. }(\mathrm{Hz})\end{array}$ & $\begin{array}{c}\text { Measured freq. } \\
(\mathrm{Hz})\end{array}$ & $\begin{array}{c}\text { Error } \\
(\mathrm{Hz})\end{array}$ \\
\hline 1 & 454.9 & 477.5 & 22.6 \\
2 & 1137.6 & 1167.5 & 29.9 \\
3 & 1802.7 & 1857.5 & 54.8 \\
4 & 2733.2 & 2805 & 71.8 \\
\hline
\end{tabular}

rotational DOFs; therefore, the whole system possesses 216 DOFs. After assembling the governing equations for all the elements and incorporating the boundary conditions, the equation of motion is written as

$$
\mathbf{M} \ddot{q}+(\mathbf{C}+\Omega \mathbf{G}) \dot{q}+\mathbf{K} q=f(t),
$$

where $q$ and $f(t)$ are generalized displacement and generalized force vector in two radical directions; $\Omega$ is the rotation speed; $\mathbf{M}, \mathbf{C}$, and $\mathbf{K}$ represent square symmetric mass, damping, and stiffness matrices, respectively; $\mathbf{G}$ is the skew symmetric gyroscopic matrix. For a free-free axisymmetric rotor under steady state condition, $\mathbf{C}$ and $\mathbf{K}$ do not include the bearing supporting damping and stiffness; $\Omega$ is zero.

The material of the rotor is a nickel-base superalloy with good corrosion resistance. The FE code compiled in MATLAB for this rotor does not contain the unknown interactions of shrink fit interfaces, inhomogeneous materials, small geometrical details, and so on, which cause potential errors to the modelling. For the rotor of this AMB system, its theoretical first four free-free bending modal frequencies and mode shapes calculated by the mathematical finite element model without model updating are listed and drawn in Table 2 and Figure 3, respectively.

3.2. Modal Test and Modal Assurance Criterion. Modal experiment is implemented to update and verify the rotor mathematical model. The rotor is suspended vertically at the end with a string. One acceleration sensor is fixed on the rotor (node 40) and an instrumented impulse hammer is employed to impact the rotor at 17 different axial locations (nodes 1, 4, 7, 11, 15, 17, 20, 23, 29, 31, 35, 38, 40, 43, 46, 52, and 54). The noise and vibration analyser and the modal analysis software are employed to perform the modal test, pictured in Figure 4. Experimental rotor free-free mode shapes are drawn in Figure 5 and experimental modal frequencies are listed in Table 2.

The modal assurance criterion $[14,15]$ is widely used to evaluate the comparison of mode shapes, which is defined as a scalar constant relating the degree of consistency (linearity) between one modal and another reference modal vector as follows:

$$
\operatorname{MAC}_{i j}=\frac{\left|\left\{\varphi_{A}\right\}_{i}^{T}\left\{\varphi_{X}\right\}_{j}\right|^{2}}{\left(\left\{\varphi_{A}\right\}_{i}^{T}\left\{\varphi_{A}\right\}_{i}\right)\left(\left\{\varphi_{X}\right\}_{j}^{T}\left\{\varphi_{X}\right\}_{j}\right)},
$$

where $\left\{\varphi_{A}\right\}_{i}$ is analytical modal vector for mode $i ;\left\{\varphi_{X}\right\}_{j}$ is compatible test modal vector for mode $j$. The resulting MAC 
TABLE 3: Comparison for MAC value.

\begin{tabular}{lcc}
\hline Bending models & MAC (initial) & MAC (updated) \\
\hline 1 & 0.998 & 0.999 \\
2 & 0.989 & 0.986 \\
3 & 0.985 & 0.984 \\
4 & 0.979 & 0.987 \\
\hline
\end{tabular}

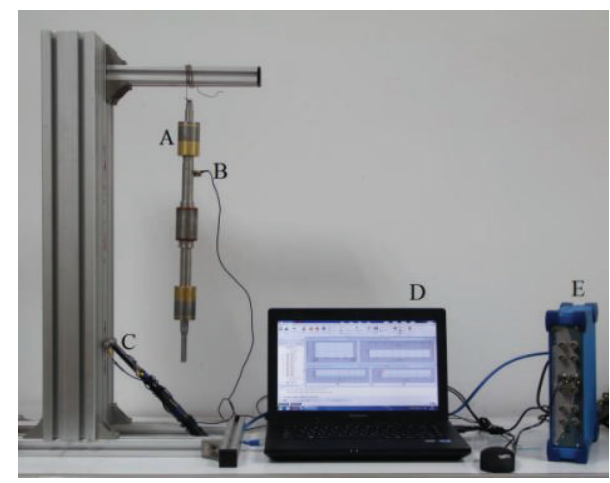

\begin{abstract}
A: Rotor of the rotor AMB system D: Computer
B: Acceleration sensor $\quad$ E: Noise and

C: Impulse hammer vibration analyser
\end{abstract}

FIGURE 4: Modal test devices.

value always lies between zero and one, with a value of one indicating a perfect match. The MAC values $(i=j)$ before model updating are listed in Table 3. Combining Tables 2 and 3 , although it is clear that the discrepancy for modal frequencies is significant, the MAC values are close to one, which indicates a good match. The reason is that although the rotor manufacture processing is complicated, the geometry property is simple, causing the MAC results to approximate to one before updating.

3.3. Model Updating. Many sources of uncertainties exist in the rotor modelling including unknown structure features and simplifications. To update the rotor finite element model so it can fit the experimental measurements, we need to select model design variables carefully. For the rotor studied here, its physical properties such as mass, geometry, and polar and transverse moments of inertia properties can be determined accurately but the rotor stiffness of some region is difficult to obtain since the front/rear radical and thrust AMB lamination stacks, sensors reference stacks and motor, and so on are shrunk fit with the rotor, which contribute to the bending stiffness. Therefore, in the rotor finite elements model, the modulus of elasticity for these uncertain areas (pictured in Figure 6) is updated. Although there are 24 beam elements in the shrink fit areas, 6 design variables are selected since the front $\mathrm{AMB}$ is the same as the rear. For the same reason, the variables for two motor rotor fix ring areas are equivalent. The model updating variables corresponding to the physical rotor are defined in Table 4. Apart from those 6 variables, the modulus of elasticity of other finite elements
TABLE 4: Model updating variables (stiffness values) corresponding to the physical rotor.

\begin{tabular}{lc}
\hline Updating variable & Description/position \\
\hline$e_{1}$ & Thrust AMB lamination stacks \\
$e_{2}$ & Radical AMB lamination stacks \\
$e_{3}$ & Sensor ring \\
$e_{4}$ & Motor fix stacks \\
$e_{5}: e_{6}$ & Motor rotor \\
\hline
\end{tabular}

(without shrink fit effect) is assumed to be accurate. Note that the variables listed in Table 4 are initially assigned with the same value using other finite elements without shrink fit effect.

Initialization of the updating process begins with the definition of an error function. Since the finite element model is different from actual system, the model updating method tries to minimize the difference. Here first four modal frequency errors and corresponding MAC are selected as updating target which are written as follows:

$$
\begin{aligned}
\varepsilon_{\omega i} & =\left|\omega(e)_{A i}-\omega_{X i}\right|, \\
\varepsilon_{M i} & =\frac{1}{\operatorname{MAC}(e)_{i i}}-1,
\end{aligned}
$$

where $\omega(e)_{A i}$ is the $i$ th theoretical modal frequency obtained through finite element analysis program; $\omega_{X i}$ is the $i$ th test modal frequency data; $\operatorname{MAC}(e)_{i i}$ is the modal assurance criteria values between the $i$ th mode shape of the finite element model and the $i$ th identified test mode shape. $\varepsilon_{\omega i}$ and $\varepsilon_{M i}$ represent the corresponding modal frequency errors and MAC values, respectively. Compared with the MAC updating target $1-\mathrm{MAC}_{i i}$ adopted from [16], the MAC updating target applied to (4) can provide high sensitivity when the MAC value is not close to one. Combining modal frequency and MAC error is necessary and could provide more advantages since if only modal frequency is selected as an error target, the modal frequency of updated model could match perfectly; however, the mode shape discrepancy may be significant, resulting in an invalid updated model, although the initial first four MAC values are close to one. Therefore, the error function is calculated in

$$
\operatorname{err}\left(\varepsilon_{\omega i}, \varepsilon_{M i}\right)=\sum_{i=1}^{4} k_{\omega i} \varepsilon_{\omega i}+\sum_{i=1}^{4} k_{M i} \varepsilon_{M i}
$$

where the terms $k_{\omega i}$ and $k_{M i}$ represent weight factors for the modal frequency error $\varepsilon_{\omega i}$ and MAC values $\varepsilon_{M i}$, which are listed in Table 5. Note that the first three weight factors are bigger since the first three modal frequencies are the most concerned, and the MAC error target $\varepsilon_{M i}$ adopted in error function provides the mode shape accuracy.

The smaller the error value, the more accurate the AMB rotor model. The essence of the minimization of $\operatorname{err}\left(\varepsilon_{\omega i}, \varepsilon_{M i}\right)$ is an optimization problem, which begins with the calculation of current model's modal frequency and mode shapes, then calculates the error between analytical and experimental data, and eventually obtains the error function $\operatorname{err}\left(\varepsilon_{\omega i}, \varepsilon_{M i}\right)$ value. 

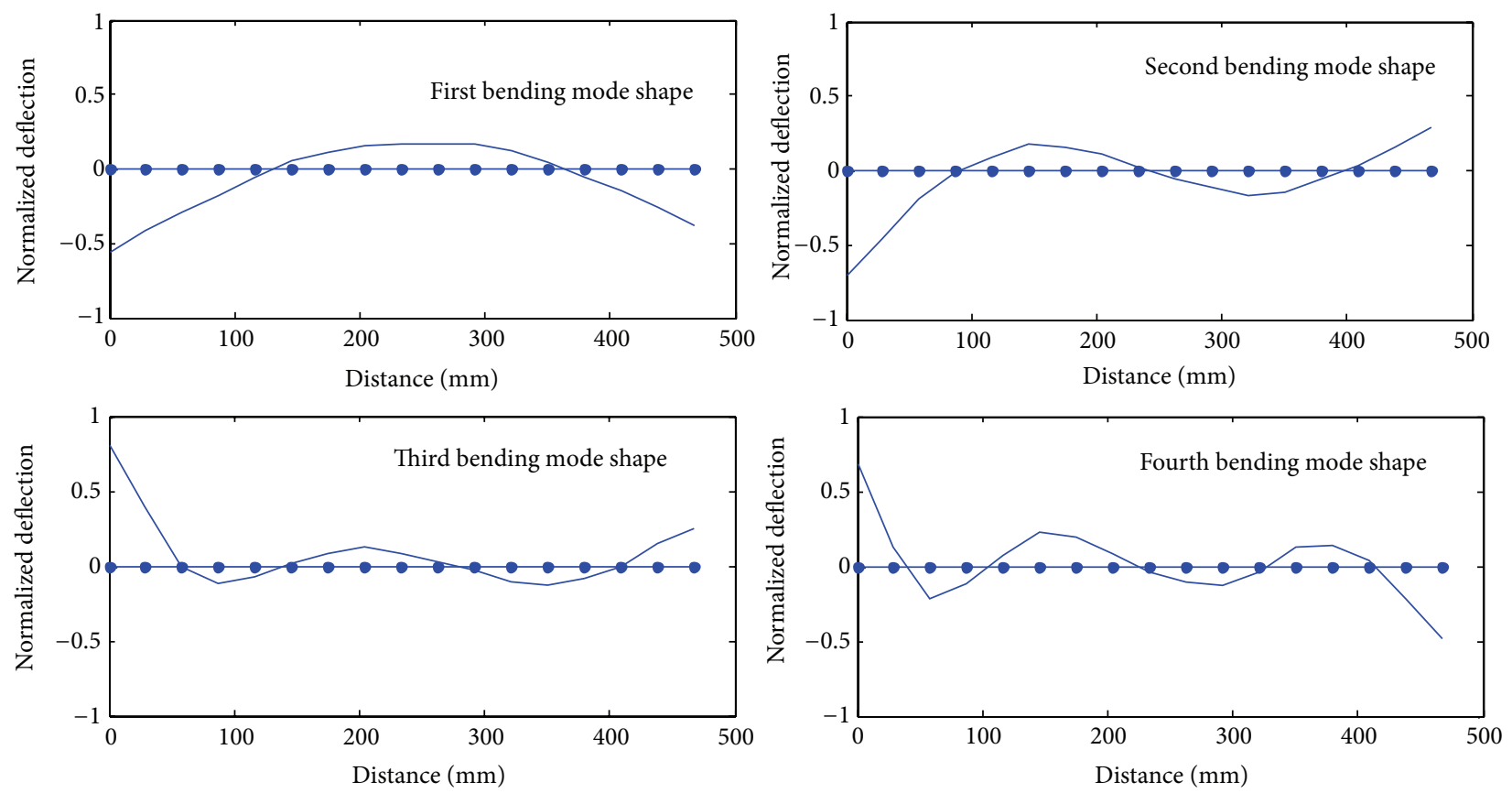

FiguRE 5: Experimental free-free rotor mode shapes.

TABLE 5: Weight factors for error targets.

\begin{tabular}{lc}
\hline Weight factor & Value \\
\hline$k_{\omega 1}$ & 8 \\
$k_{\omega 2}$ & 3 \\
$k_{\omega 3}$ & 2 \\
$k_{\omega 4}$ & 1 \\
$k_{M 1}: k_{M 4}$ & 1 \\
\hline
\end{tabular}

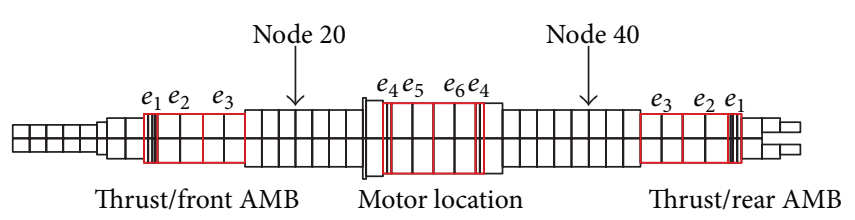

FIGURE 6: FE rotor model indicating locations of the 6 moduli of elasticity design variables.

Under the optimization routine, another prospective $e$ is generated for the next loop evaluation. The minimization stops until the criterion is met.

Optimization of the error function is an important step in the model updating. The Nelder-Mead simplex method is an optimization algorithm solving the multidimensional nonlinear problem, which is employed in this paper. It was first proposed by Nelder and Mead [17]. This optimization method adopts each peak value of a function to find the function trend and provides a basis to find a method making function descend. The simplex [18-20] refers to a polyhedron that has $n+1$ peaks in an $n$-dimensional space. When we use simplex method to search optimization solutions, the simplex is updated through a progression of reflection, expansion, and contraction operations, where the one of the updated simplex vertices, at least, is smaller than the original simplex.

For an optimization problem involving $n$ variables, the simplex method searches for an optimal solution by evaluating a set of $n+1$ points (i.e., points forming a simplex), denoted as $x_{1}, x_{2}, \ldots, x_{n+1}$. The $n+1$ vertices need to satisfy $f\left(x_{1}\right) \leq f\left(x_{2}\right) \leq \cdots \leq f\left(x_{n+1}\right)$. For the AMB rotor adopted in this paper $n=6$, and there will be 7 points, where each point contains 6 stiffness variables; the $f$ stands for the error function $\operatorname{err}\left(\varepsilon_{\omega i}, \varepsilon_{M i}\right)$ in (5).

Firstly, replace the worst point $x_{n+1}$ with a new point $x_{r}$ generated by reflecting $x_{n+1}$ over the centroid of the remaining points

$$
x_{r}=\bar{x}+\alpha\left(\bar{x}-x_{n+1}\right),
$$

where

$$
\bar{x}=\frac{1}{N}\left(x_{1}+x_{2}+\cdots+x_{n}\right) ;
$$

$\alpha$ is the reflection coefficient; evaluate $f_{r}=f\left(x_{r}\right)$.

Second, if the reflected point $x_{r}$ looks promising (i.e., smaller than the smallest point in the current simplex, $f_{r}<$ $f_{1}$ ), a new point further along the reflection direction is generated using the equation

$$
x_{e}=\bar{x}+\gamma\left(\bar{x}-x_{n+1}\right),
$$

where $\gamma$ is called the expansion coefficient $(\gamma>1)$ because the resulting simplex is expanded.

Third, if the reflected point $x_{r}$ is bigger than the biggest point in the original simplex (i.e., $f_{r} \geq f_{n+1}$ ), a new point 
TABLE 6: Comparison for modal frequencies.

\begin{tabular}{lccc}
\hline $\begin{array}{l}\text { Bending } \\
\text { models }\end{array}$ & $\begin{array}{c}\text { Updated } \\
\text { freq. }(\mathrm{Hz})\end{array}$ & $\begin{array}{c}\text { Measured freq. } \\
(\mathrm{Hz})\end{array}$ & Error $(\mathrm{Hz})$ \\
\hline 1 & 477.5 & 477.5 & 0 \\
2 & 1167.5 & 1167.5 & 0 \\
3 & 1855.4 & 1857.5 & 1.1 \\
4 & 2827.7 & 2805 & 22.7 \\
\hline
\end{tabular}

close to the centroid on the same side of $x_{n+1}$ is generated using the equation

$$
x_{c}=\bar{x}-\beta\left(\bar{x}-x_{n+1}\right),
$$

where $\beta$ is called the contraction coefficient $(0<\beta<1)$ because the resulted simplex is contracted.

Fourth, if the reflected point $x_{r}$ is smaller than $x_{n+1}$ but is bigger than the second worst point $x_{n}$ in the original simplex (i.e., $f_{n} \leq f_{r} \leq f_{n+1}$ ), a new point close to the centroid on the opposite side of $x_{n+1}$ is generated using the equation

$$
x_{c}=\bar{x}+\beta\left(\bar{x}-x_{n+1}\right) \text {. }
$$

After the steps above, the method forms a new simplex by replacing the worst $x_{n+1}$ point in the former simplex. The iteration will be terminated when the error is below the criterion

$$
\left\{\frac{1}{n} \sum_{i=1}^{n}\left[f\left(x_{i}\right)-f(\bar{x})\right]\right\}^{1 / 2} \leq \varepsilon
$$

where $\varepsilon$ represents the standard error set in advance.

The basic idea of the simplex method is to use the simplex peak to calculate the peak function values and compare them with each other. Then it finds a favourable search direction, step length, and a better point to replace the inferior peak point, which produces a new simplex to replace the original simplex. Thus, the simplex will continuously approach the minimum value of the objective function until it finds the minimum point.

After about 1500 iterations, an adequate solution is obtained, which are plotted in Figure 7 (red line is the original value). The updated modal frequency and MAC results are compared in Tables 6 and 3, showing near zero error in terms of the first three modal frequencies and a perfect mode shape match. The difference for the fourth mode frequency is slightly big since the weight factor for the fourth mode frequency error is relatively small given that the first modal frequencies draw our most interest. Although the MAC values in terms of 2 nd and 3rd bending models drop slightly, they are still very close to one. Note that the MAC updating target adopted in the error function equation (5) has high sensitivity when its value is close to zero but has low sensitivity when the value is close to one, which guarantees that the mode shape is correct as well as the modal frequency.

\section{Updated Model Verification}

Verification of the updated rotor AMB model is achieved by comparing the rotor analytical and experimental frequency

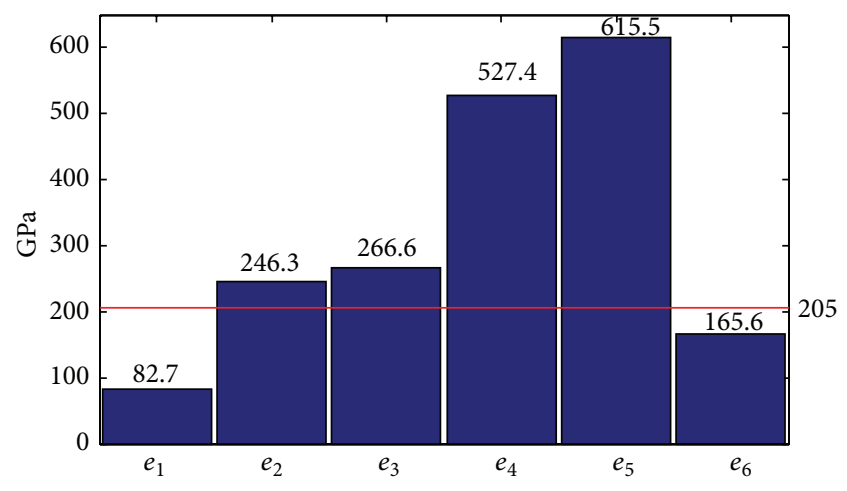

FIGURE 7: The modulus of elasticity values before and after updating.

response functions (FRFs). For the model FRFs calculation, a proportional damping can be assumed as $\mathbf{C}=\delta \mathbf{K}+$ $\varphi \mathbf{M}$, where $\delta$ and $\varphi$ are real scalars. In order to make the analytical frequency response peaks match the corresponding experimental peaks, we need proper values of $\delta$ and $\varphi$. In the modal test, the modal damping ratio $\zeta_{i}$ for $i$ th mode can be obtained through the modal analysis software directly. In the present experiment, $\zeta_{1}=0.15 \%, \zeta_{2}=0.07 \%, \zeta_{3}=0.12 \%$, and $\zeta_{4}=0.17 \%$. One set value of $\delta$ and $\varphi$ can be obtained using the following [21]:

$$
2 \zeta_{i} \omega_{i}=\delta+\varphi \omega_{i}^{2},
$$

where $\omega_{i}$ is the modal frequency $(\mathrm{rad} / \mathrm{s})$. Because one set of $\delta$ and $\varphi$ can be calculated using two different $\zeta_{i}$, using the four damping ratios above, six sets of results are obtained and the average of these data is adopted to calculate the analytical FRF. The specific input and output points between node 20 and node 40 are shown in Figure 6.

Figure 8 illustrates the comparison between nonupdated model and experimental frequency response. It is clearly shown that, without model updating, the difference between the two frequency responses is quite evident and the modelling error becomes more significant in the high frequency range. However, the frequency response of the resulting updated rotor $\mathrm{AMB}$ model matches the experimental frequency response quite well in the range from 0 to $3000 \mathrm{~Hz}$ as shown in Figure 9. Both the system resonance and antiresonance frequencies are accurate for the first three modes. The model is less accurate in the high frequency range due to the selected weighting factors and assumptions made for beam elements in this model. Some of the antiresonance frequencies shifting may be caused by the experimental errors such as an inconsistent or inaccurate location where the hammer impact is added.

\section{Conclusion}

The rotor dynamic properties play a key role in the rotor $\mathrm{AMB}$ system. In order to obtain an accurate rotor mathematical model, model updating techniques are often employed to update the unconstrained model. Generally, models are 

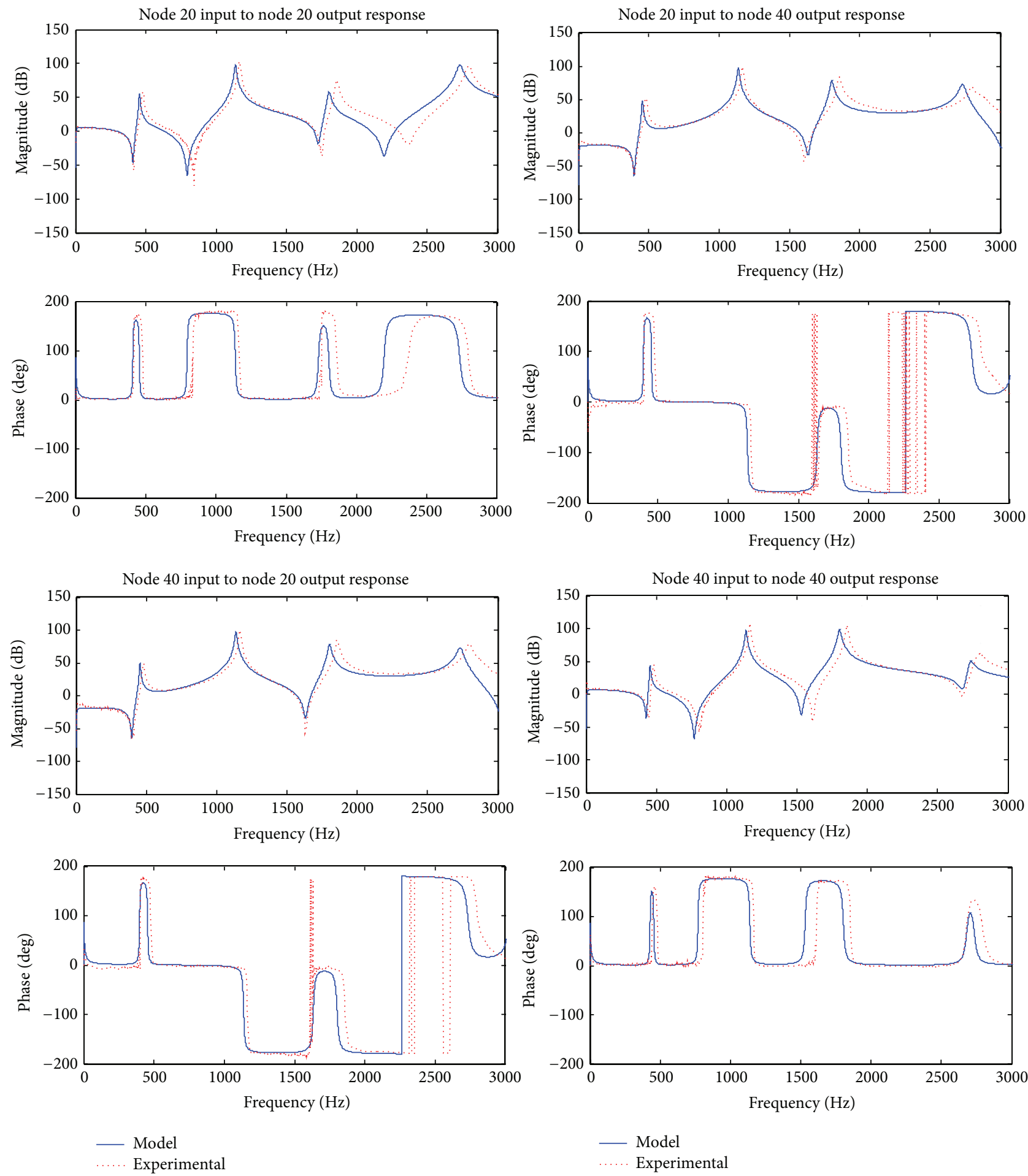

FigURE 8: Experimental and nonupdated model frequency response comparison.

corrected by applying an appropriate error function in terms of resonance frequencies; however, mode shape accuracy has been shown to be insufficient. In this paper, a model updating approach considering both resonance and mode shape is proposed and the mode shape error target adopted in this paper provides high sensitivity when the MAC value is not close to one. The interference fits and shrink effects on the rotor shaft are considered during the variable selection and Nelder-Mead simplex method is employed to optimize the FE rotor model. In order to make sure the updated model agrees with the actual rotor, the FRFs comparison between updated model response and experimental data is performed. 

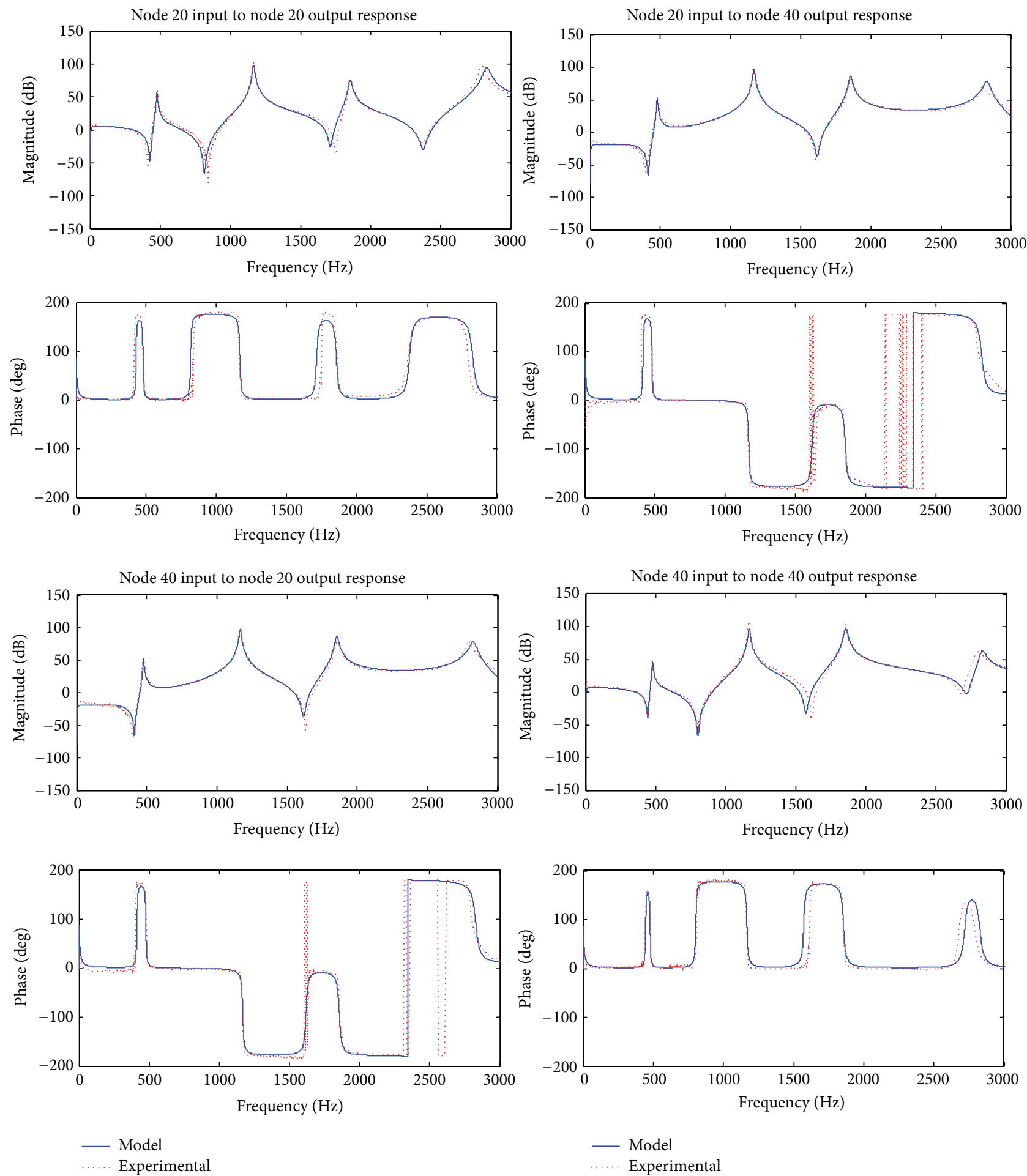

FIGURE 9: Experimental and updated model frequency response comparison.

The results show that the updated theoretical model is fairly accurate compared to experimental data, which indicates the updating method proposed in this paper is feasible for the rotor AMB system.

\section{Conflict of Interests}

The authors declare that there is no conflict of interests regarding the publication of this paper. 


\section{Acknowledgment}

This research has been supported by the Natural Science Foundation of China (51275240, 51205186).

\section{References}

[1] G. Schweitzer and E. H. Maslen, Magnetic Bearings: Theory, Design, and Application to Rotating Machinery, Springer, New York, NY, USA, 2009.

[2] G. Li, Z. Lin, P. E. Allaire, and J. Luo, "Modeling of a high speed rotor test rig with active magnetic bearings," Journal of Vibration and Acoustics, vol. 128, no. 3, pp. 269-281, 2006.

[3] A. C. Wroblewski, J. T. Sawicki, and A. H. Pesch, "Rotor model updating and validation for an active magnetic bearing based high-speed machining spindle," Journal of Engineering for Gas Turbines and Power, vol. 134, no. 12, p. 122509, 2012.

[4] A. Lanzon and P. Tsiotras, "A combined application of $H_{\infty}$ loop shaping and $\mu$-synthesis to control high-speed flywheels," IEEE Transactions on Control Systems Technology, vol. 13, no. 5, pp. 766-777, 2005.

[5] L. Di and Z. Lin, "Control of a flexible rotor active magnetic bearing test rig: a characteristic model based all-coefficient adaptive control approach," Control Theory and Technology, vol. 12, no. 1, pp. 1-12, 2014.

[6] G. C. Horner and W. D. Pilkey, "The Riccati transfer matrix method," Journal of Mechanical Design, vol. 100, no. 2, pp. 297302, 1978.

[7] A. C. Wroblewski, Model identification, updating, and validation of an active magnetic bearing high-speed machining spindle for precision machining operation [Doctoral dissertation], Cleveland State University, 2011.

[8] J. Vance, F. Zeidan, and B. Murphy, Machinery Vibrations and Rotordynamics, John Wiley \& Sons, 2010.

[9] A. Berman and W. G. Flannelly, "Theory of incomplete models of dynamic structures," AIAA Journal, vol. 9, no. 8, pp. 1481-1487, 1971.

[10] M. Friswell and J. E. Mottershead, Finite Element Model Updating in Structural Dynamics, vol. 38, Springer, 1995.

[11] M. Chouksey, J. K. Dutt, and S. V. Modak, "Model updating of rotors supported on journal bearings," Mechanism and Machine Theory, vol. 71, pp. 52-63, 2014.

[12] J. E. Mottershead and M. I. Friswell, "Model updating in structural dynamics: a survey," Journal of Sound and Vibration, vol. 167, no. 2, pp. 347-375, 1993.

[13] N. M. M. Maia and J. M. M. Silva, "Modal analysis identification techniques," Philosophical Transactions of the Royal Society of London. Series A: Mathematical, Physical and Engineering Sciences, vol. 359, no. 1778, pp. 29-40, 2001.

[14] R. J. Allemang, "The modal assurance criterion-twenty years of use and abuse," Sound and Vibration, vol. 37, no. 8, pp. 14-23, 2003.

[15] M. Pastor, M. Binda, and T. Harčarik, "Modal assurance criterion," Procedia Engineering, vol. 48, pp. 543-548, 2012.

[16] B. A. Zárate and J. M. Caicedo, "Finite element model updating: multiple alternatives," Engineering Structures, vol. 30, no. 12, pp. 3724-3730, 2008.

[17] J. A. Nelder and R. Mead, "A simplex method for function minimization," The Computer Journal, vol. 7, no. 4, pp. 308-313, 1965.
[18] J. Sun and Y. Liang, Optimization of Mechanical Design, China Machine Press, 2006.

[19] J. Yen, J. C. Liao, B. Lee, and D. Randolph, "A hybrid approach to modeling metabolic systems using a genetic algorithm and simplex method," IEEE Transactions on Systems, Man, and Cybernetics Part B: Cybernetics, vol. 28, no. 2, pp. 173-191, 1998.

[20] J. C. Lagarias, J. A. Reeds, M. H. Wright, and P. E. Wright, "Convergence properties of the Nelder-Mead simplex method in low dimensions," SIAM Journal on Optimization, vol. 9, no. 1, pp. 112-147, 1998.

[21] I. Chowdhury and S. P. Dasgupta, "Computation of Rayleigh damping coefficients for large systems," The Electronic Journal of Geotechnical Engineering, vol. 8, 2003. 

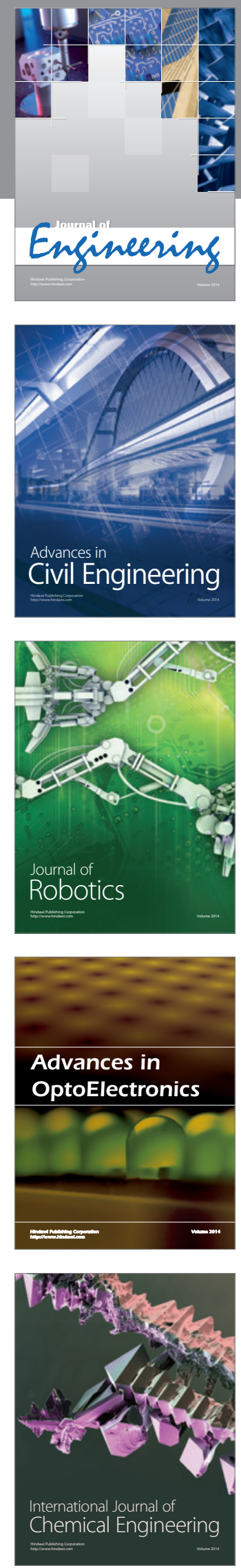

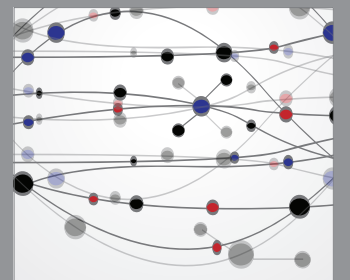

The Scientific World Journal
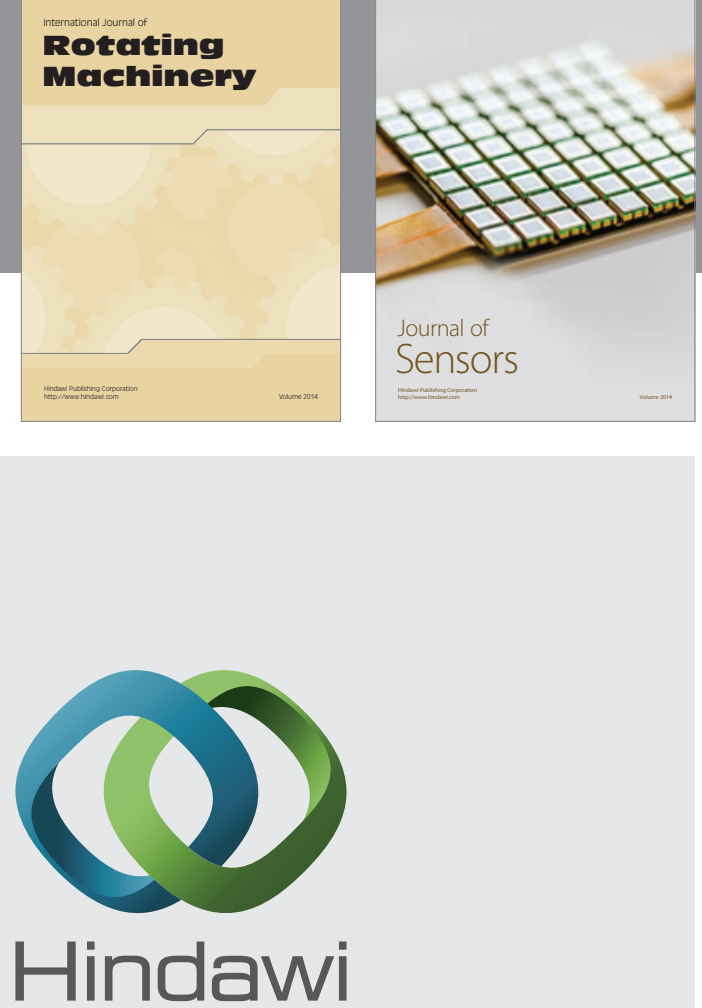

Submit your manuscripts at http://www.hindawi.com
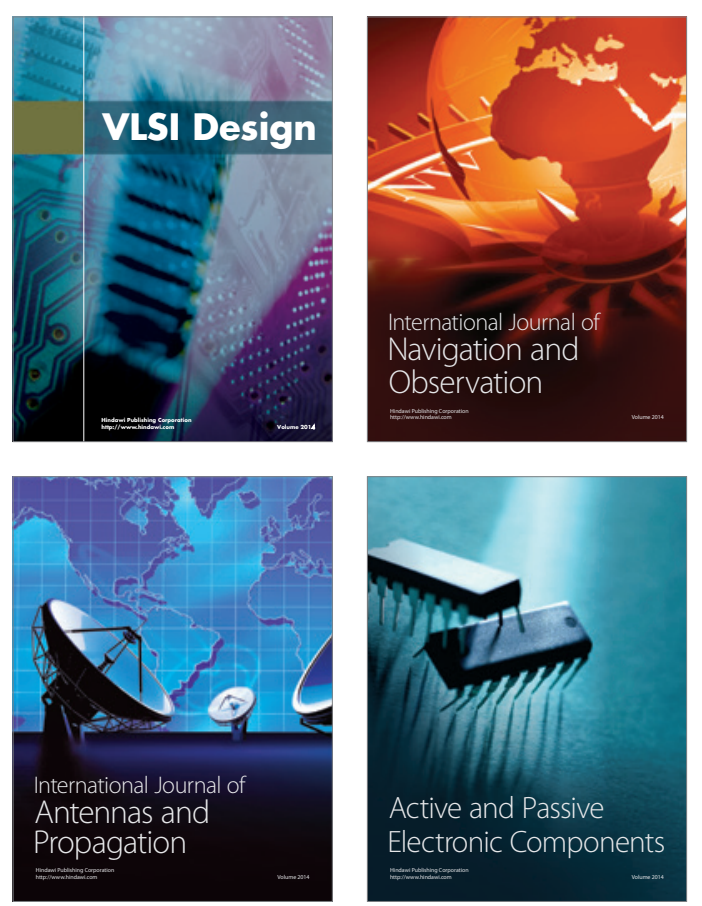
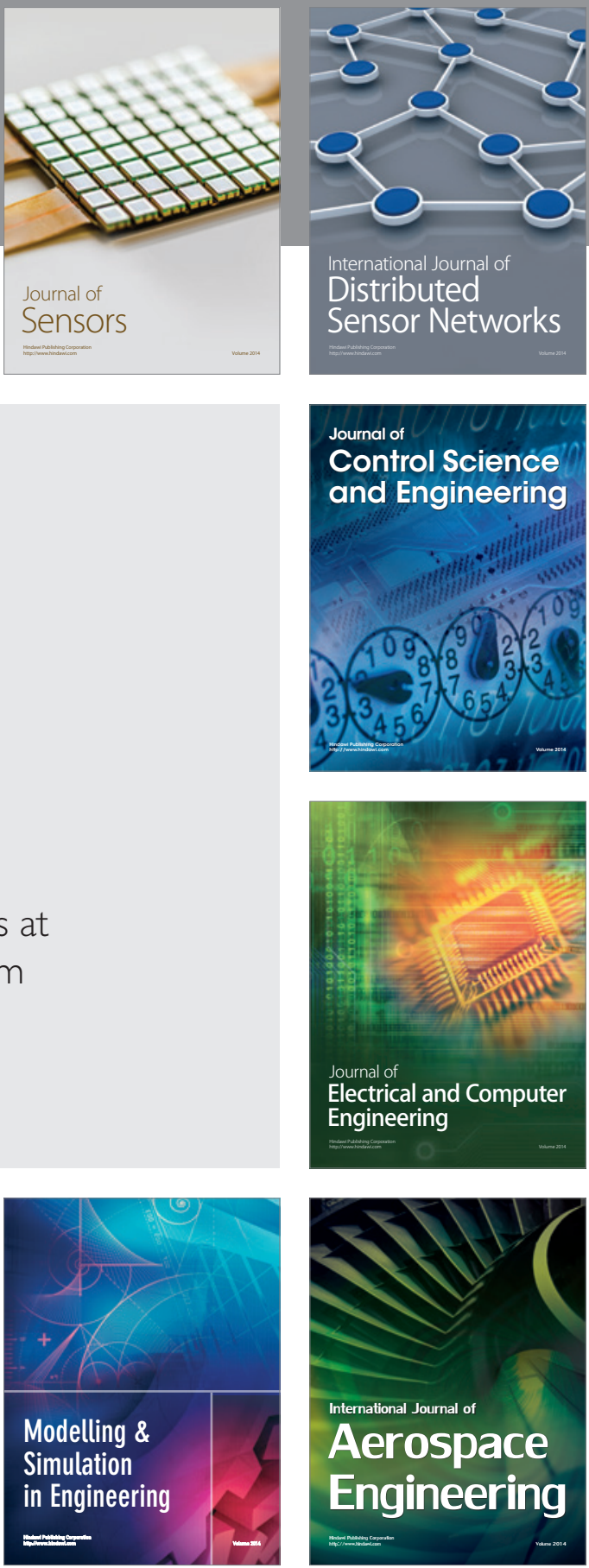

Journal of

Control Science

and Engineering
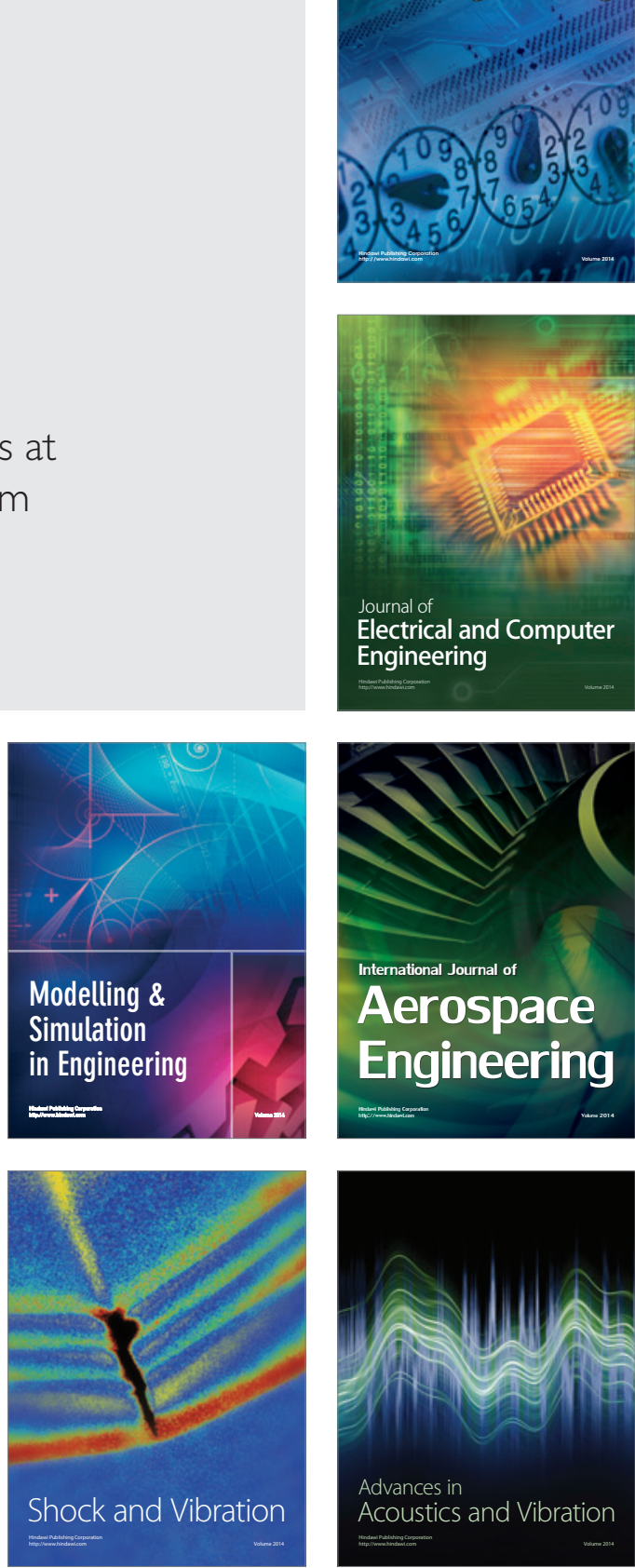